\title{
PHILOSOPHI ... ERRAVERUNT (SPEC. UNIV. 1. 41) DER TRAKTAT DE ANIMA IM SPECULUM UNIVERSALE DES RADULFUS ARDENS
}

\author{
EIN BEMERKENSWERTES BEISPIEL FÜR KRITISCHE REZEPTION \\ DER PLATONISCHEN SEELENLEHRE IN DER FRÜHSCHOLASTIK
}

\begin{abstract}
Summary: In the late twelfth century Platonic philosophy is gaining more and more importance for western thought in general, in particular for Christian theology. As part of this "Renaissance of the twelfth century" a new perspective is emerging, focusing on the reality of man and nature. In this context the Speculum uniuersale of Radulfus Ardens is an interesting example, which has, however, hardly been investigated. In his opus (containing 14 books) Radulfus Ardens constructs a virtue-ethical framework, for the first time connecting the morality of man systematically with anthropological facts and psychology. Radulfus Ardens receives the Platonic theory of soul and criticizes it in order to integrate some of its elements into his ethical concept. His main concern is a holistic perspective on man. The aim of this essay is to present an Early Scholastic concept of soul theory by comparing the Platonic theory of soul with the explanations of Radulfus Ardens in the first book of his Speculum uniuersale. Thus, I try to shed light on an important phase of the reception of Plato in medieval western thought.
\end{abstract}

Key words: soul theory, parts oft he soul, virtue ethics, Radulfus Ardens, Speculum uniuersale

\section{VORHABEN, UNTERSUCHUNGSGEGENSTAND UND VERFAHREN}

Die folgende Untersuchung beabsichtigt, einen in der Forschung unzureichend untersuchten Zeitraum der Platonrezeption - das letzte Drittel des 12. Jahrhunderts - anhand des Werkes eines bisher weitgehend unbeachteten Autors - dem Speculum uniuersale ${ }^{1}$ des Radulfus Ardens, dem umfangreichsten tugendethischen Entwurf des 12. Jahrhun-

${ }^{1}$ Das Speculum uniuersale (abgekürzt: Spec. uniu.) ist in neun Handschriften zugänglich, von denen vier den vollständigen Text überliefern, die übrigen jedoch nur einzelne Bücher oder Exzerpte enthalten. Eine kritische Edition der Bücher 1-5 liegt inzwischen vor: Radulfi Ardenti Speculum uniuersale. Ed. C. HeimANN - S. ERnST [CCCM 241]. Turnhout 2011, 3-451. In der Einleitung der Edition (XXXVIII-CVIII) findet sich auch eine ausführliche Beschreibung der Handschriften. Vgl. dazu z. B. auch ERNST, S.: Die passiones animae im Speculum universale des Radulfus Ardens. In Passiones animae. Die "Leidenschaften der Seele" in der mittelalterlichen Theologie und Philosophie. Ein Handbuch. Hrsg. C. SCHÄFER - M. THURNER. Berlin 2013², 135. 
derts $^{2}-$ zu untersuchen. Den Untersuchungsgegenstand wird dabei in erster Linie die Rezeption von bestimmten Elementen aus der Seelenlehre Platons innerhalb des ersten Buches des Speculum uniuersale (gelegentlich auch als Traktat De anima bezeichnet) ${ }^{3}$ darstellen.

Damit ist das Ziel der Untersuchung benannt: Unter Beleuchtung des klar umgrenzten Bereiches der Seelenlehre wird zum einen das schriftstellerische Werk eines Denkers in Augenschein genommen, der an der Schwelle zum 13. Jahrhundert unmittelbar vor dem Einsetzen der intensiven Aristotelesrezeption ein in sich geschlossenes Konzept einer Tugendethik unter Rückgriff auf die pagan-antike Philosophie entwirft. ${ }^{4}$ Zum anderen werden exemplarisch der Stellenwert und die Verwendung der platonischen Philosophie sowie der Umgang mit speziellem platonischem Gedankengut am Übergang von der Früh- zur Hochscholastik genauer betrachtet. Eine strengen Maßstäben genügende quellenkritische Untersuchung des Speculum uniuersale kann in diesem Rahmen nicht unternommen werden, ebenso wenig eine umgreifende Beleuchtung der Platonrezeption im 12. Jahrhundert.

Dieser Zielsetzung folgend soll deshalb zunächst in aller gebotenen Kürze ein Überblick über die Rolle der platonischen Philosophie vor dem Hintergrund der geistesgeschichtlichen Entwicklung im 12. Jahrhundert geboten werden, um sodann zu begründen, warum die Wahl speziell auf das Werk des Radulfus Ardens gefallen ist. Im Anschluss daran ist es notwendig, sich für die im Zentrum des Beitrags gelegene Untersuchung der Texte des Radulfus Ardens die wichtigsten Aspekte der platonischen Seelenlehre ins Gedächtnis zu rufen, um abschließend zu resümieren, in welcher Form und aus welchem Grund im Speculum uniuersale platonische Motive auffindbar sind und welche Bedeutung sie für seinen Gesamtansatz haben.

\section{DIE ROLLE DER PLATONISCHEN PHILOSOPHIE IM 12. JAHRHUNDERT UND DIE GRÜNDE FÜR DIE AUSWAHL DES SPECULUM UNIVERSALE DES RADULFUS ARDENS}

Hohes Interesse an der paganen antiken - und dabei besonders der platonischen Philosophie stellt im 12. Jahrhundert einen entscheidenden Bereich des geistigen Auf-

\footnotetext{
${ }^{2}$ Vgl. ERnST, S.: Klug wie die Schlangen und ehrlich wie die Tauben. Die Lehre von den Komplementärtugenden als Strukturprinzip der Tugendlehre des Radulfus Ardens. MthZ 61 (2010) 43.

${ }^{3}$ Vgl. Michaud-QuAntin, P.: Die Psychologie bei Radulfus Ardens, einem Theologen des ausgehenden 12. Jahrhunderts. MThZ 9.2 (1958) 81-96.

${ }^{4}$ Das Werk des Radulfus Ardens lässt die differenzierte und breitgefächerte Kenntnis antiker Philosophie des Autors erkennen. Im Speculum uniuersale werden namentlich v. a. Platon, Aristoteles, Seneca und Cicero genannt, wobei von Aristoteles nur die Schriften Metaphysica, De anima und der Categoriae, nicht jedoch die Ethica Nicomachea als bekannt gelten dürfen (vgl. GRÜNDEL, J.: Die Lehre des Radulfus Ardens von den Verstandestugenden auf dem Hintergrund seiner Seelenlehre. München et al. 1976, 51 sowie ERNST, S.: «Estote prudentes sicut serpentes et simplices sicut columbae» - der Gedanke der Komplementärtugenden im Speculum uniuersale des Radulfus Ardens. In Spiritus et Littera. Beiträge zur Augustinus-Forschung. Festschrift Cornelius Mayer OSA. Hrsg. G. FÖRSTER - A. GROTE - C. MüLLER [Cass. 39.6]. Würzburg 2009, 552-556); Freilich spielen auch die Schriften der Neuplatoniker Boëthius, des Macrobius und des Porphyrios eine wichtige Rolle.
} 
bruchs zu neuen Ufern dar; in vielen Bereichen ${ }^{5}$ zeigt sich eine vorher ungekannte Aufnahme von griechisch-römischem Gedanken- und Kulturgut, weshalb man nach einem Terminus von Charles Homer Haskins auch von der „Renaissance of the Twelfth Century ${ }^{\prime 6}$ spricht. Angestoßen durch das Bestreben Anselms von Canterbury zu Beginn der Scholastik an der Schwelle vom 11. zum 12. Jahrhundert, den christlichen Glauben von innen heraus unter Zuhilfenahme der Vernunft, genauer zu erhellen, bemüht sich eine Vielzahl von Gelehrten im Umfeld der „Kathedralschulen“, 7 Offenbarung und tradierte kirchliche Lehre mit Hilfe von bestimmten antiken philosophischen Begriffen, Motiven und Denkrichtungen zu erläutern sowie eine Synthese aus Beidem herzustellen.

Dass dabei vor allem auf Elemente platonischer Provenienz zurückgegriffen wird, hat verschiedene Gründe. Zunächst ist die Literatur der Kirchenväter - allen voran das einflussreiche Euvre Augustins sowie die Schriften des Pseudo-Dionysius Areopagita - in weiten Teilen vom Platonismus geprägt. Daher wird die platonische und neuplatonische Philosophie im Gegensatz zu den Lehren des Aristoteles allein schon vom traditionellen Standpunkt aus als mit dem christlichen Denken kompatibel angesehen. ${ }^{8}$ Zweitens greifen die Gelehrten jener Zeit aber auch aus bestimmten thematischen Präferenzen zu den wenigen im griechischen Original verfügbaren PlatonDialogen. Die Gelehrten der „Kathedralschulen“ - wobei hier in besonderem Maße die Vertreter der „Schule“ von Chartres zu nennen sind - entwickeln eine völlig neue Sicht auf die Eigenwirklichkeit der Natur und des Menschen; in der Forschung spricht man daher vom Aufkommen einer scientia naturalis, in deren Rahmen man bestrebt ist, bestimmte Phänomene und natürliche Gegebenheiten unter Zuhilfenahme der Vernunft bzw. der Philosophie zu erklären. ${ }^{9} \mathrm{Da}$, wie es Clemens Baeumker treffend her-

${ }^{5}$ Nicht nur in den Wissenschaften (vgl. Zimmermann, A.: Die Theologie und die Wissenschaften. In Die Renaissance der Wissenschaften im 12. Jahrhundert. Hrsg. P. WEIMAR. Zürich 1981, 87-105 sowie KluXEN, W.: Der Begriff der Wissenschaft. In Die Renaissance der Wissenschaften im 12. Jahrhundert. Hrsg. P. WEIMAR. Zürich 1981, 273-293), sondern auch im Bereich der Kunst, Architektur, Ökonomie und Technik kommen bedeutsame Neuerungen auf. Dies zeigt sich auch am Anwachsen der Städte, die mit den entstehenden „Kathedralschulen“ den Kristallisationspunkt des geistigen Lebens bilden (vgl. dazu die Übersicht in ERNST, S.: Ethische Vernunft und christlicher Glaube. Der Prozess ihrer wechselseitigen Freisetzung. In der Zeit von Anselm von Canterbury bis Wilhelm von Auxerre [BGPhMA 25]. Münster 1996, 6-12.

${ }^{6}$ HASKInS, C. H.: The Renaissance of the Twelfth Century. Cambridge, Mass. 1927, vi.

${ }^{7}$ Der Begriff „Schule“ wird in Anführungszeichen gesetzt, da er einerseits nicht Schulen in unserem heutigen Sinne meint, sondern eher eine geistige Strömung, die von einem bestimmten „Lehrer“ (im Falle des Radulfus Ardens ist hier hauptsächlich auf Gislebertus Porretanus (Gilbert von Poitiers) zu verweisen, nach dem die „Porretanerschule“ benannt wurde) und/oder einem bestimmten Ort (z. B. Poitiers) ausgeht und bei mehreren „Schülern“ auffindbar ist und es andererseits um die Existenz dieser „Schulen“ einen wissenschaftlichen Disput gibt (vgl. dazu KoBusch, T.: Die Philosophie des Hoch- und Spätmittelalters. München 2011, 24; ERNST: Klug [Anm. 2] 68).

${ }^{8}$ Vgl. dazu grundlegend BEIERWALTES, W.: Platonismus im Christentum [PhA 73]. Frankfurt am Main 20012 , 7-24 sowie im Bereich der Seelenlehre THÜMmEL, H. G.: Die Seele im Platonismus und bei den Kirchenvätern. In $\psi v \chi \eta ்$, Seele, anima. Festschrift für Karin Alt. Hrsg. J. HolzHAUSEN. Stuttgart Leipzig 1998, 243-254.

${ }^{9}$ Vgl. SPEER, A.: Die entdeckte Natur. Untersuchungen zu Begründungsversuchen einer ,scientia naturalis " im 12. Jahrhundert [STGMA 45]. Leiden - New York - Köln 1995, 1-17. Zu den Schwierigkeiten, welche die Rede von einem „Platonismus“ im Mittelalter aufwirft vgl. HIRSCHBERGER, J.: 
ausgearbeitet hat, humanistisch-ästhetische und naturwissenschaftlich-medizinische Fragen dominieren, zieht man vor allem Platons Lehren heran - Aristoteles ist in jener Zeit lediglich als Logiker hauptsächlich über Boëthius bekannt. ${ }^{10}$

Da sich die literarische und ideengeschichtliche Landschaft, die sich um die genannten Problemkomplexe ausformt, als bunt, unsystematisch und schwer durchschaubar erweist, ist es kaum möglich, klare Linien der Platonrezeption auszumachen. Zusätzlich ist es nur in sehr seltenen Fällen möglich, tatsächlich herauszufinden, auf welchen platonischen Denker der jeweilige mittelalterliche Gelehrte sich beruft oder gar, welche konkrete Schrift er herangezogen hat. Vielmehr ist eine methodische $\mathrm{Zu}-$ sammenarbeit aus Philosophie bzw. Theologie und Philologie erforderlich, um die oft unter der Oberfläche versteckten und eklektisch verarbeiteten Motive ausfindig zu machen. ${ }^{11}$ Dazu möchte unsere Untersuchung im Blick auf die platonische Lehre von den Seelenvermögen - ein in der Frühscholastik im Übrigen selten verarbeiteter Stoff - einen Beitrag leisten. Dass die Wahl dabei auf das Speculum uniuersale des Radulfus Ardens fällt, scheint aus drei Gründen lohnenswert:

(i) Erstens ist der Autor dem Kreis der Porretaner - damit sind die Schüler des Gilbert von Poitiers, der für seine innovative und umstrittene Kommentierung der theologischen Traktate des Boëthius bekannt wurde - zuzuordnen. Dieser Gelehrtenkreis zeichnet sich durch eigenständige und originelle Verarbeitung philosophischen Gedankengutes aus; wir können also bei Radulfus Ardens, der als weitgehend eigenständig arbeitender Denker der zweiten Generation des Porretanerkreises angesehen werden kann, ${ }^{12}$ eine relativ fundierte Kenntnis philosophischer Schriften voraussetzen, was auch die bisherige Arbeit an der quellenkritischen Erschließung des Speculum uniuersale durch Johannes Gründel ${ }^{13}$ und die bereits erwähnte kritische Edition der Bücher 1-5 unter Beweis stellt.

Platonismus und Mittelalter. In Platonismus in der Philosophie des Mittelalters. Hrsg. W. BEIERWALTES [WdF 197]. Darmstadt 1969, 56-63.

${ }^{10}$ Vgl. BAEUMKer, C.: Der Platonismus im Mittelalter. In Platonismus in der Philosophie des Mittelalters. Hrsg. W. BEIERWALTES [WdF 197]. Darmstadt 1969, 8-10.

${ }^{11} \mathrm{Da}$ zum Einen in Bezug auf verschiedene Fragestellungen verschiedene Aspekte der platonischen Philosophie steinbruchartig herangezogen wurden und zum Anderen platonisches Gedankengut und platonische Schriften zugleich im Abendland wieder entdeckt wurden, aber auch aus Byzanz und dem Orient Westeuropa erreichten, wird in der Forschung auch von den „Platonismen des Mittelalters“ gesprochen (vgl. den gleichnamigen Beitrag von CHENU, M.-D.: Die Platonismen des XII. Jahrhunderts. In Platonismus in der Philosophie des Mittelalters. Hrsg. W. BeIERWALTES [WdF 197]. Darmstadt 1969, 268-316 sowie ERLER, M.: Plato. Hrsg. H. FlASHAR [Ueberweg.Antike 2/2]. Basel 2007, 531).

${ }^{12}$ Halfen, R.: Chartres 4. Die Kathedralschule und ihr Umkreis. Stuttgart 2011, 253. Gilbert von Poitiers selbst hat kein eigenes ethisches Konzept entwickelt; dies tun erst seine Schüler, die seine Ideen weiter denken und ihre Terminologie stark an Gilberts Werke anlehnen. Dies sind in erster Generation Alanus von Lille, Simon von Tournai sowie Johannes von Salisbury und in zweiter Generation Radulfus Ardens. Daneben gibt es noch die sog. „kleine Schule“ (vgl. DONDAINE, A.: Ecrits de la petite école Porretaine. Montreal 1962).

${ }^{13}$ Gründel hat in zwei Schriften einen maßgeblichen Beitrag zur quellenkritischen und interpretatorischen Erschließung des Speculum uniuersale geliefert (GRÜNDEL, J.: Die Lehre von den Umständen der menschlichen Handlungen im Mittelalter. Münster 1963; GRÜNDEL: Die Lehre [Anm. 4]) sowie ein vollständiges Quästionenverzeichnis des Werkes angefertigt, das eine aufschlussreiche Übersicht über die behandelten Themen ermöglicht (GRÜNDEL, J.: Das >Speculum Universale des Radulfus Ardens. München 1961). 
(ii) Radulfus Ardens entfaltet zweitens in den 14 Büchern des Speculum uniuersale einen tugendethischen Entwurf, der sich eindeutig von den mehrheitlich heilsgeschichtlich orientierten zeitgenössischen Ansätzen absetzt. Er rückt also nicht nur die von Gott gnadenhaft geschenkte Tugend der caritas in den Vordergrund, sondern stellt auch die Beteiligung des Menschen beim Tugenderwerb heraus. Es ist ihm anscheinend in Anlehnung an die im Umfeld der Schule von Chartres entstehende scientia naturalis daran gelegen, den ganzen Menschen in seiner Eigenwirklichkeit in den Blick zu nehmen. ${ }^{14}$ Damit ist er im Ringen um die Integration philosophischer Inhalte in die Theologie gleichsam einer ,,progressiven“ Gruppe von Gelehrten zuzuordnen.

(iii) Drittens spielt das erste Buch, welches wie erwähnt auch gelegentlich als Traktat De anima bezeichnet wird und die differenzierte Seelenlehre des Radulfus Ardens entwirft, eine zentrale Rolle für das Gesamtwerk. Auf der Basis der Seelenvermögen entwickelt Radulfus Ardens das Kernstück seiner Tugendethik, ${ }^{15}$ das System der Komplementärtugenden, das im Verlauf dieser Untersuchung noch exemplarisch dargestellt wird. Die Seelenlehre ist also aufs engste mit der Fähigkeit des Menschen zum sittlichen Handeln verknüpft und stellt damit die anthropologische Grundlage der Tugendethik dar. Radulfus Ardens wird daher auch als einer der Begründer des psychologischen Ansatzes in der Tugendethik angesehen, den später Thomas von Aquin (wenn auch auf anderem Fundament) zur vollen Entfaltung bringen wird. ${ }^{16}$

Unser Autor verspricht also mit dieser Gewichtung der Lehre von der Seele und ihren Vermögen einen fruchtbaren Gegenstand für die Suche nach platonischem Gedankengut. Bevor wir die Sache selbst angehen, seien die tragenden Elemente der platonischen Seelenlehre umrissen.

\section{GRUNDZÜGE DER IM 12. JAHRHUNDERT EINFLUSSREICHEN ASPEKTE DER PLATONISCHEN SEELENLEHRE}

Gleich zu Beginn dieser äußerst knappen Darstellung sei gesagt, dass der Frage, welche platonischen Schriften in welcher Form in der Zeit des Radulfus Ardens zur Verfügung standen, nicht nachgegangen wird. Der Grund dafür besteht darin, dass es sich hier, wie bei der Erläuterung der Methodik gefordert, um eine rein motivgeschichtliche Untersuchung handelt. Freilich ist es bedeutsam, welche Inhalte der platonischen Lehre von den Seelenvermögen im 12. Jahrhundert überhaupt verfügbar waren; dabei ist einerseits auf die im Original zugänglichen Dialoge Phaidon und Timaios $^{17}$ zu verweisen, andererseits waren Einzelaspekte der Politeia durch die Wer-

\footnotetext{
${ }^{14}$ Vgl. ERNST: Estote prudentes (Anm. 4) 323-333; ERNST: Klug (Anm. 2) 46-50; ERNST: Die passiones (Anm. 1) 136-143.

${ }^{15}$ Dass eine komplementäre Denkstruktur die systematische Grundlage der Tugendlehre des Radulfus Ardens darstellt, hat zuerst ERNST: Estote prudentes (Anm. 4) 333-338 herausgestellt.

${ }^{16} \mathrm{Vgl}$. MichaUd-QuANTIN (Anm. 3) 93-94.

${ }^{17}$ Zur Verfügbarkeit des Timaios in der Frühscholastik vgl. GIBSON, M.: The Study of the 'Timaeus' in the Eleventh Century. Pensamiento 25 (1969) 183-194.
} 
ke der Kirchenväter und Florilegiensammlungen bekannt. Daraus lässt sich in wie in einem Brennspiegel zusammenfassen: ${ }^{18}$

(i) Der Phaidon geht von der Eingestaltigkeit (vgl. Phaid. 78b-80e) der menschlichen Seele ( $\psi v \chi \eta ์)$ aus und legt damit den Schwerpunkt auf das vernünftige Seelen-

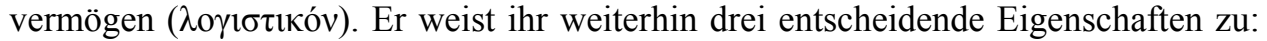
Sie liegt jeglicher Form des Lebens zugrunde und ist das Prinzip aller Bewegung (vgl. dazu auch Phaidr. 245c-246a) ${ }^{19}$; daher belebt und beseelt sie auch den menschlichen Körper $(\sigma \tilde{\omega} \mu \alpha)$. Sie stammt aus der Ideenwelt, strebt daher nach dem Guten, Wahren und Schönen und ist präexistent (vgl. Phaid. 77b). Durch ihren Fall aus der Ideenwelt sind ihre Aufgaben die Wiedererinnerung (àvó $\mu v \eta \sigma ı)$ ) und das Abstreifen der weltlichkörperlichen Verunreinigungen. Die Seele ist damit die eigentliche Seinsgrundlage des Menschen und ist im Gegensatz zum Körper ewig und unvergänglich (vgl. Phaid. 65b); ihre Aufgabe besteht darin, mithilfe der Vernunft den Menschen im diesseitigen Leben zu leiten. Damit wird die Bedeutung des sittlich guten Lebens und der Tugenden herausgestellt und an die Seelenlehre angebunden. Schließlich ist sie unvergänglich und mit der Idee des Lebens verknüpft.

(ii) Die Politeia entwickelt besonders unter staatstheoretischen und ethischen Aspekten die Seelenlehre weitaus differenzierter und z. T. auch im Widerspruch zu den früheren Dialogen. Sie führt neben dem vernünftigen Seelenvermögen (in der Forschung ist diesbezüglich auch von Seelenteilen die Rede) noch das muthafte bzw.

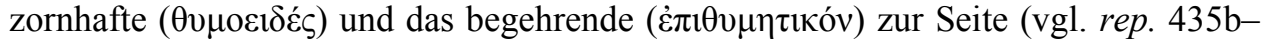
441c). Die Vernunft ist zuständig für das Unterscheiden, der Mut bzw. der Zorn für das Streben bzw. den Ärger und das Begehren für die Lusterfahrung. Der Vernunft obliegt die Leitung der beiden anderen, die sich als ihr als wertvolle Hilfsmittel erweisen können (vgl. rep. 441a). Es ist Aufgabe des Menschen, die drei Seelenvermögen, die in Unordnung geraten können, miteinander in Einklang zu bringen und zu harmonisieren; damit gewichtet Platon das Gefühlsleben des Menschen deutlich stärker und schwächt den Intellektualismus seines Lehrers Sokrates ab.

(iii) Die Dichotomie der Seelenlehre der Politeia greift der Timaios auf, der von einem sterblichen (dem mut- bzw. zornhaften und dem begehrenden) und einem unsterblichen (dem vernünftigen) Seelenteil spricht. Im Zentrum dieses Dialogs steht die vom Demiurgen erschaffene Weltseele, die alle Geschicke im Universum lenkt und mit der die Seele des einzelnen Menschen verknüpft ist (vgl. Tim. 27c-37c; 41de); dabei stellt die Weltseele das Urbild und die einzelne Menschenseele das Abbild dar. ${ }^{20}$ Damit wird noch einmal in aller Deutlichkeit die Mittlerfunktion der Seele zwischen dem transzendenten, aber durch die Schöpfung aber auch immanenten Demiurgen und der Welt selbst herausgestellt. Der Timaios war im Umfeld der Kathedralschulen eine häufig gelesene Schrift und wurde durch das hohe Interesse an natur-

\footnotetext{
${ }^{18}$ Folgende Kurzübersicht richtet sich neben den ausgewiesen Quellentexten aus Platons Werken besonders nach ERLER (Anm. 11) 375-390 sowie ERLER, M.: Platon. München 2006, 133-142.

${ }^{19}$ Vgl. BARTH, H.: Die Seele in der Philosophie Platons. Tübingen 1921, 284-321. 1992,98 .

${ }^{20}$ Vgl. z. B. Steiner, M. P.: Psyche bei Platon [Neue Studien zur Philosophie 3]. Göttingen
} 
philosophischen Fragestellungen im Rahmen der Entstehung der bereits erwähnten scientia naturalis vielfältig rezipiert. ${ }^{21}$

Dass das Konzept der platonischen Seelenlehre keineswegs einheitlich und letztlich auch nicht systematisierbar ist, sei hier nur am Rande erwähnt. ${ }^{22}$ Dieses Problem war den mittelalterlichen Gelehrten einerseits bewusst und sie griffen gezielt die Elemente heraus, die zur Erhellung theologischer Fragen hilfreich erschienen, andererseits waren die meisten Inhalte (wie bereits erwähnt) ohnehin aus zweiter oder dritter Hand und in verflachter Form zugänglich. Eine diffuse Zusammensetzung aus den hier angedeuteten Elementen der drei genannten Schriften dürfte wohl den Wissensstand der philosophisch gebildeten Zeitgenossen des Radulfus Ardens grob umreißen.

\section{DARSTELLUNG DER SEELENKONZEPTION DES RADULFUS ARDENS UNTER DER PERSPEKTIVE IHRER BEDEUTUNG FÜR SEINEN TUGENDETHISCHEN ANSATZ}

Innerhalb des ersten Buches des Speculum uniuersale entwickelt Radulfus Ardens seine Seelenlehre in insgesamt 17 Kapiteln (Spec. uniu. 1. 38-54). Alleine die Tatsache, dass er diese Ausführungen am Anfang seines Werkes positioniert, verdient besondere Beachtung. Es war in der theologischen Literatur keineswegs üblich und vermutlich sogar unausgesprochen untersagt, eigens Untersuchungen über die Seele anzustellen. Bei den wenigen Gelehrten, die sich mit dieser Thematik beschäftigen, stehen naturwissenschaftlich-medizinische Fragestellungen im Vordergrund. Diese werden meist in einem gesonderten Traktat abgehandelt und sind nicht in einen solch umfangreichen Entwurf wie das 14 Bücher umfassenden Speculum uniuersale eingebettet. $^{23}$ Obgleich die genannten 17 Kapitel innerhalb des Werkes einen recht überschaubaren Raum einnehmen, scheinen sie also allein schon von der Positionierung her von entscheidender Bedeutung zu sein. Um diese klarer herauszustellen, werden zuerst einige maßgebliche Kapitel genauer betrachten, um dann deren Ertrag in Beziehung zu der bereits erwähnten Lehre von den Komplementärtugenden zu setzen.

Radulfus Ardens eröffnet die Thematik in Kapitel 38 mit der Feststellung, dass die Tugenden zum einen durch die göttliche Gnade geschenkt werden und zum ande-

${ }^{21}$ Freilich war der Dialog nicht in Form des griechischen Originals zugänglich, sondern hauptsächlich durch den Kommentar des Chalkidius aus dem 4. Jahrhundert sowie durch eine weniger weit verbreitete lateinische Übersetzung Ciceros (vgl. GIBSON [Anm. 17] 183-184).

${ }^{22}$ Vgl. dazu im Allgemeinen ERLER: Plato (Anm. 11) 378 sowie GuTHRIE, K. C. W.: Plato's Views on the Nature of the Soul. In Recherches sur la tradition platonicienne. Ed. GUTHRIE, K. C. W. et al. [EnAu 3]. Vandœuvres-Genève 1955, 3-22 und speziell in Bezug auf die Bestandteile und die Ein-

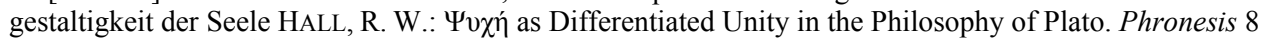
(1963) 63-82. Eine Übersicht über verschiedene Lösungsversuche sowie einen schlüssigen, von der stetigen Weiterentwicklung der Seelenlehre im Euvre Platons ausgehenden Ansatz liefert GROAG, E.: Platons Lehre von den Seelenteilen. WS 35. 2 (1914) 321-352.

${ }^{23} \mathrm{Vgl}$. MiCHAUD-QUANTIN (Anm. 3) 92-93. 
ren aus den Seelenvermögen selbst entstehen. ${ }^{24}$ Damit ist nicht nur der Fokus von der traditionellen heilsgeschichtlichen Konzeption der Tugendethik - die an dieser Stelle im Übrigen weder große Beachtung findet, noch eingehend wiederlegt wird - auf die Eigenwirklichkeit des Menschen verschoben, sondern es wird auch im Verweis auf die Seelenlehre eine anthropologische Grundlegung des vorgestellten Ansatzes in Aussicht gestellt.

Sodann werden in Kapitel 39 die Eigenschaften der Seele genannt: Sie ist zunächst vernunftbegabt. Sodann ist sie eine unsterbliche Substanz, die dem Feuer in ihrer Bewegung nach oben (also zu Gott hin) gleicht. ${ }^{25}$ Diese bis hier genannten Attribute stellen deutlich heraus, dass es sich bei der Seele um ein organum handelt, das einer höheren Sphäre entstammt und selbst wieder dorthin ausgerichtet ist. Der Begriff substantia betont dabei, dass sie nicht ein Wesenselement des Menschen unter vielen ist, sondern für den Menschen, seine Lebensaufgabe und seine Ausrichtung auf Gott hin die entscheidende Instanz seiner Existenz schlechthin darstellt. Diese Wertschätzung der Seele wird auch in den späteren Büchern des Speculum uniuersale dadurch unterstrichen, dass Radulfus Ardens Körper und Seele als Antipoden ansieht und sich anscheinend stark an monastisch-asketischen Idealen orientiert, was u. a. seine vielen Beispiele aus den Vitae patrum unter Beweis stellen. ${ }^{26}$ Weiterhin führt Radulfus Ardens an, dass die Seele den Körper belebt und - für unsere Untersuchung eine höchst interessante Aussage - die Sinne zum Begehren bewegt, womit er dem Intellektualismus und einer rein heilsgeschichtlich orientierten Tugendkonzeption eine deutliche Absage erteilt.

Auf der Grundlage dieser wichtigen Vorbemerkungen geht unser Autor direkt im Anschluss in den Kapiteln 40 und 41 auf die Vermögen der Seele bzw. ihrer Kräfte genauer ein. Weil die Seele nämlich von Gott dazu geschaffen ist, zwischen Gut und Böse zu unterscheiden, um auf diese Weise das höchste Gut zu erkennen und zu erlangen, ${ }^{27}$ ist sie mit verschiedenen Vermögen bzw. Kräften ausgestattet: mit der natürlichen Vernunft (ratio), mit den beiden affektiven Vermögen, nämlich des Begehrens bzw. Liebens (concupiscibilitas oder amabilitas) einerseits und des Zürnens bzw. Hassens (irascibilitas oder odibilitas) andererseits sowie mit der Verfügungsgewalt (potestas) und der Freiheit (libertas) ${ }^{28}$ - die beiden letztgenannten sind zwar für den

${ }^{24}$ Spec. uniu. 1. 38: Quoniam ex diuina auctoritate uirtutes oriri, iam et ex parte demonstratum est et in sequentibus plenius exponetur. Nunc ex qua origine uel ex quorum adminiculo oriantur, demonstrandum est. Que quoniam oriuntur originaliter ex proprietatibus anime, ne incerta per incertiora demonstrentur, de anima eiusque proprietatibus aliquid uel breuiter insinuandum est [...].

${ }^{25}$ Spec. uniu. 1. 39: Est igitur anima substantia rationalis et immortalis, motu ignea, organum membrorum uiuificando corpori et mouendis auide sensibus attributa. [...] Anima uero ad instar ignis semper sursum ad suum principium tendit.

${ }^{26}$ In diesem Zusammenhang ist erwähnenswert, dass Radulfus Ardens (wohl aus didaktischer und protreptischer Motivation) häufig Episoden aus den Vitae patrum anführt, um die von ihm dargestellten theoretischen Inhalte zu veranschaulichen (vgl. dazu den Index und den Quellenapparat der in Anm. 1 angeführten kritischen Edition).

${ }^{27}$ Spec. uniu. 1. 40: Voluit quippe deus talem creare animam, que inter bonum malumque discerneret, summum bonum intelligeret [...].

${ }^{28}$ Spec. uniu. 1. 41: Eapropter dedit ei naturalem rationem cum comitibus suis, concupiscibilitatem quoque et irascibilitatem siue odibilitatem cum sequelis suis, potestatem quoque et libertatem. 
Gesamtentwurf des Radulfus Ardens wichtig, haben für unsere Untersuchung aber nur untergeordnete Bedeutung.

Die in diesem Abschnitt bereits vorweggenommenen entscheidenden Begrifflichkeiten verdienen nun eine genauere Betrachtung. Zunächst greift Radulfus Ardens in Bezug auf die ersten drei Seelenvermögen eindeutig platonische Termini auf, nämlich ratio, concupiscibilitas und irascibilitas, wobei er sogar eigens auf die Autorität der philosophi verweist, allerdings ohne genauer auszuführen, auf wen er sich denn nun tatsächlich bezieht - eine in der gesamten Scholastik weit verbreitete Verfahrensweise. Freilich unterzieht er seine Gewährsmänner sogleich der Kritik; mit der nicht gerade zurückhaltend gewählten Wendung errauerunt bezichtigt er die gesamte platonische Tradition in einem für sie charakteristischen Bereich des Irrtums. ${ }^{29}$ Eine Begründung für diese Aussage bleibt er dem Leser nicht schuldig: irascibilitas und concupiscibilitas, also Zorn und Begehren, werden der ganzheitlichen Ausrichtung der Seele auf das Gute nicht gerecht. Zornig sein und begehren kann man aber nur hinsichtlich gegenwärtiger und zukünftiger, nicht aber vergangener Dinge. Er wirft den Philosophen also vor, mit diesen Begrifflichkeiten die Art an die Stelle der Gattung gestellt zu haben und führt sogleich seiner Ansicht nach besser geeignete Termini ein, nämlich odibilitas und amabilitas, also Liebe und Hass. Er verweist dabei darauf, dass Liebe und Hass grundlegendere Bestimmungen der menschlichen Affektivität als Zorn und Begehren darstellen. ${ }^{30}$ Diese Änderung mag als Nebensächlichkeit oder gar als unbedeutende Kleinigkeit erscheinen - tatsächlich liegt hier in nuce das Kernstück der Tugendlehre des Radulfus Ardens verborgen und zwar in zweierlei Hinsicht:

(i) Zum einen führt uns der Magister vor Augen, dass zur Ausrichtung des Lebens am Guten nicht alleine die Vernunft ausreicht, sondern dass vielmehr ein motivierendes Moment hinzutreten muss und zwar in der Gestalt von Liebe (also Erstreben des Guten) und Hass (also Abwehr des Schlechten); damit sind natürlich auch die Hoffnung auf Lohn und die Furcht vor Strafe verbunden (vgl. Spec. uniu. 1. 40). Radulfus Ardens macht deutlich, dass weder allein das Vernunfturteil, noch allein der affektive Impuls das Handeln des Menschen in rechter Weise leiten kann; ${ }^{31}$ vielmehr gilt es, beide in einer Ordnung, aufeinander zu beziehen, die einerseits von Natur aus vorgegeben, aber andererseits bei jedem einzelnen Menschen spezifisch ausgeformt werden muss. ${ }^{32}$ Dies gelingt durch die Ausformung der Tugenden. ${ }^{33}$ Die Komponenten

${ }^{29}$ Spec. uniu. 1. 41: Tamen philosophi pro odibilitate irascibilitatem minus qui proprie posuerunt, qui uolentes ostendere tres aptitudines anime dixerunt eam naturaliter rationalem, concupiscibilem et irascibilem, sed in appellatione duarum extremarum aptitudinum errauerunt.

${ }^{30}$ Spec. uniu. 1. 41: [...] nam omnis concupiscibilitas est amabilitas, non autem econuerso. Concupiscimus enim tantum nondum habita, amamus uero tam non habita quam habita. Concupiscimus quoque tantum presentia uel futura, amamus uero preterita et presentia et futura.

${ }^{31}$ Spec. uniu. 1. 42: Enimuero nisi anima ratione predita esset, nequaquam bonum discernere aut queritare posset, nisi quoque cum ratione etiam concupiscibilitatem et odibilitatem ceterasque affectiones cum potestate et libertate possideret, nec bonum nec malum promereri nec premio nec pena remunerari ualeret. Quomodo enim promereri ualeret, que neque bene uelle nec male posset? Quomodo quoque remunerari ualeret, que nec gaudere nec tristari posset?

${ }^{32}$ Spec. uniu. 1. 46: Habent autem predicte uires et affectiones quemdam naturaliter ordinem, necessitatem et inequilitatem. [...] Enimuero sicut nichil prodest discernere sine affectacione, sic nichil pro- 
der amabilitas und der odibilitas müssen dabei unter Leitung der Vernunft so in Einklang miteinander gebracht werden, dass sich jeweils aus dem Vermögen des Liebens und dem Vermögen des Hassens eine Tugend ausbildet, welche die jeweils andere im Gleichgewicht hält. Diese Systematik findet sich schon in Bezug auf die Tugenden des vernünftigen Seelenteils (uirtutes discretiuae) in den Büchern 7-10. ${ }^{34}$ Dort verbindet Radulfus Ardens mehr oder weniger pragmatisch-assoziativ verschiedene Tugenden und Laster miteinander, z. B. im neunten Buch die Klugheit (prudentia) mit der Einfachheit (simplicitas), um nur ein Beispiel zu nennen. Im Bereich derjenigen Tugenden, die aus den beiden einander entgegengesetzten bzw. einander ergänzenden Vermögen der amabilitas und der odibilitas heraus entstehen, also in den Büchern 11 und 12, ergänzen sich jeweils eine uirtus amatiua und eine uirtus oditiua, sodass die beiderseitige Ergänzung gleichsam in der Struktur der Seele grundgelegt ist. Zur Veranschaulichung dieser Konzeption sei beispielhaft auf das komplementäre Verhältnis spes und timor aus dem elften Buch verwiesen: Die Komplementärtugend der Hoffnung (spes) ist die Furcht (timor). Diese beiden halten sich im Gleichgewicht; wenn jedoch eine der beiden Seiten zu sehr überbetont wird, verwandelt sich die Tugend der spes in das Laster der Vorwegnahme (presumptio) und die Tugend des timor in das Laster der Verzweiflung (desperatio). ${ }^{35}$ Das folgend aufgeführte Schaubild veranschaulicht diese Systematik nochmals:

(ii)

\begin{tabular}{|c|c|c|c|}
\hline \multicolumn{2}{|c|}{ amabilitas } & \multicolumn{2}{|c|}{ odibilitas } \\
\hline $\begin{array}{c}\text { uitium } \\
\text { presumptio }\end{array}$ & $\begin{array}{c}\text { uirtus collateralis } \\
\text { spes } \rightarrow\end{array}$ & $\begin{array}{c}\text { uirtus collateralis } \\
\leftarrow \text { timor }\end{array}$ & $\begin{array}{c}\text { uitium } \\
\text { desperatio }\end{array}$ \\
\hline
\end{tabular}

(iii) Zum anderen ist es ein fundamentales Anliegen des Radulfus Ardens, die Welt und besonders den Menschen im Ganzen zu betrachten. In diesem Zusammenhang ist auch die zunächst nebensächlich erscheinende Bemerkung zu verstehen, dass die herkömmliche Terminologie sichtlich nur die Seelenregung gegenüber gegenwärtigen oder zukünftigen, nicht aber vergangenen Dingen im Blick habe. Diese gleichsam enzyklopädische Vorgehensweise findet sich im Übrigen vielerorts im Speculum uniuersale und zwar nicht nur in Hinsicht auf inhaltliche, sondern auch strukturelle Aspekte. Entscheidend ist, dass Radulfus Ardens damit klar in das geistige Umfeld der Porretaner einzuordnen ist, deren Bestreben sich ja zusammenfassend in einem ver-

dest affectare sine discretione. Inequalitatem quoque frequenter habent, quoniam alii magis discernunt quam affectant, alii magis affectant quam discernunt, alii satis eque discernere uidentur et affectare.

${ }^{33}$ Spec. uniu. 1. 47: Porro ex potentiarum siue uirium recto usu oriuntur specialiter omnes ille uirtutes, que nos instruunt, dirigunt et moderantur.

${ }^{34}$ Eine systematische Darstellung der einzelnen uirtutes discretiuae findet sich bei GRÜNDEL: Die Lehre (Anm. 4) 290-372.

${ }^{35}$ Spec. uniu. 1. 28-29: Porro spei contraria est desperatio. Et sicut spes non omnis est bona, sic et desperatio non omnis est mala. [...] Male uero spei que est uel in uirtute uel in sapientia uel in potentia hominis, opposita est bona desperatio ab eis. Sicut enim malum est in uirtute nostra uel in meritis nostris sperare, sic bonum est de uirtute nostra et de meritis nostris desperare et in solo Deo sperare. [...] Collateralis uero uirtus spei est timor. 
stärkten Interesse an der Eigenwirklichkeit der Natur und ihrer Betrachtung auf Basis des christlichen Glaubens mithilfe der platonischen Philosophie skizzieren lässt.

In Radulfus Ardens haben wir also einen Denker vor uns, der einerseits klar antik-philosophische Motive aufgreift, ohne dass freilich seine Gewährsmänner eindeutig zu ermitteln wären; quellenkritische Untersuchungen lassen die Verwendung des Werkes Liber de anima des Arabers Auicenna vermuten. ${ }^{36}$ Andererseits gibt er sich nicht mit der reinen Wiedergabe zufrieden, sondern denkt bestimmte Elemente eigenständig weiter und integriert sie in seinen Gesamtentwurf.

\section{PLATONISCHES GEDANKENGUT IN DER SEELENLEHRE DES RADULFUS ARDENS - SECHS ZUSAMMENFASSENDE THESEN UND IHR ERTRAG}

Auf dieser Grundlage gilt es abschließend zu resümieren, inwieweit man von der Verarbeitung platonischer Inhalte und Motive in Bezug auf die Seelenlehre des Speculum uniuersale sprechen kann. Im Folgenden sollen die sieben wichtigsten Vergleichspunkte thesenartig zusammengestellt werden:

(i) Bei Radulfus Ardens ist ganz wie bei Platon die Seele und ihre Struktur sowie ihre Ausrichtung auf das summum bonum elementare Grundlage für die Fähigkeit des Menschen zum moralischen Handeln.

(ii) Der Mensch wird nach Rückkehr in die Sphäre, der er durch den Besitz seiner Seele entstammt und die er wiederum ausgerichtet ist, nach moralischen Maßstäben gemessen. Im Zuge dessen erhält er Lohn (premium) oder Strafe (pena).

(iii) Wie in der platonischen Philosophie ist die Seele nicht nur ein organum unter vielen. Sie ist auch nicht wie in der aristotelischen Gedankenwelt nur die Form des Menschen, die mit dem Körper in beinahe gleichrangigem Verhältnis steht, sondern macht den ,wahren Menschen“ aus.

(iv) Die Seele ist die Instanz im Menschen, von der alle sinnlichen und geistigen Wahrnehmungen ausgehen, um sich dort wieder zu bündeln und verarbeitet $\mathrm{zu}$ werden. Der Körper ist dabei nur Hülle und Werkzeug bzw. noch Antipode des wahren Menschen in Gestalt der Seele.

(v) Die Seelenvermögen bzw. -kräfte entfalten die Tätigkeit der eingestaltigen Seele nach verschiedenen Richtungen hin. Es handelt sich also nicht nur - wie bei Aristoteles, auf dessen Seelenlehre die Forschung bisher in Zusammenhang mit

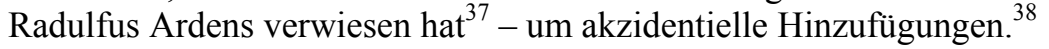

(vi) Die Terminologie der drei ersten Seelenvermögen, dem vernünftigen, dem muthaften und dem begehrenden, ist aus dem Platonismus entlehnt, wobei Radulfus

${ }^{36}$ Im Quellenapparat von Spec. ипiu. 1. 41 wird auf Auicenna Anim. 4. 4 und 5. 7 verwiesen.

${ }^{37}$ Vgl. GRÜNDEL: Die Lehre (Anm. 4) 104-105.

${ }^{38} \mathrm{Zu}$ den Einzelheiten der aristotelischen Seelenlehre vgl. den aufschlussreichen Überblick bei SHIELDS, C.: 29. Die Seele. In Aristoteles-Handbuch. Leben - Werk - Wirkung. Hrsg. C. RAPP - K. CORCILIUS. Stuttgart-Weimar 2011, 313-323. 
Ardens noch zwei weitere hinzufügt und die platonischen Motive in seinem Sinne umarbeitet.

(vii) Radulfus Ardens verankert wie Platon das Gefühlsleben und die Affekte des Menschen in der Seele in ihrer Funktion als Wesenselement der menschlichen Existenz. Er weist ihnen bei dem Streben nach dem summum bonum und dem Erwerb der Tugenden einen Anteil zu.

Es soll freilich am Ende unserer Untersuchung trotz dieser auffälligen Gemeinsamkeiten nicht ungesagt bleiben, dass es sich hier um eine Skizze in groben Zügen handelt und manche Aspekte kaum beleuchtet werden konnten. So weist Radulfus Ardens der Gefühlswelt als motivierendes Moment des Menschen eine weitaus bedeutendere Stellung als Platon ein, für den diese in bestimmten Dialogen lediglich minderwertige Seelenteile darstellen oder schlichtweg überhaupt keine Rolle spielen. Außerdem finden sich im Speculum uniuersale sieben und nicht nur drei Seelenvermögen; es kommen nämlich noch die Freiheit (libertas) und die Macht zu handeln (potentia) sowie die belebende Kraft (uegetatio) und die Sinnlichkeit (sensualitas) hinzu, die sich bei Platon nicht finden.

Ungeachtet dessen verdienen die Ergebnisse in folgendem Zusammenhang Beachtung: Radulfus Ardens entwirft an einem bisher wenig untersuchten Wendepunkt der europäischen Geistesgeschichte einen tugendethischen Ansatz, der durch inhaltliche und literarische Aspekte bereits ins 13. Jahrhundert - das Jahrhundert der Universitäten und des Aristoteles - verweist, zugleich aber von der geistesgeschichtlichen Grundlage und der Methodik noch fest im 12. Jahrhundert - dem Jahrhundert der Kathedralschulen und Platons - verankert ist. ${ }^{39}$ Dabei verarbeitet er in seinem Werk an entscheidender Stelle platonisches Gedankengut und arbeitet es letztlich völlig eigenständig in seinen Gesamtentwurf ein. Das Speculum uniuersale stellt somit einerseits ein bemerkenswertes Bindeglied zwischen zwei Epochen dar, deren Grenzen freilich nur schemenhaft wahrnehmbar sind, andererseits steht es für eklektische und selbstständige Fruchtbarmachung platonischer Philosophie in einer Originalität, die bisher noch kaum Beachtung gefunden hat.

\author{
Tobias Janotta \\ Julius-Maximilians-Universität Würzburg \\ Katholisch-Theologische Fakultät \\ Institut für Systematische Theologie \\ Lehrstuhl für Moraltheologie \\ 97070 Würzburg \\ Deutschland \\ tobias.janotta@uni-wuerzburg.de
}

${ }^{39}$ Dazu Michaud-QuANTin (Anm. 3) 94, der sich mit der Frage beschäftigt, welche Bedeutung dem Speculum uniuersale zukommt, obgleich es in der Folgezeit kaum rezipiert wurde: „Seine Isolierung ist übrigens nur relativ, sie zeigt tatsächlich eine der letzten Anstrengung, eine Lehre de anima vor der Einführung der aristotelischen Psychologie zu konzipieren. Die Einführung einer solchen Darstellung in ein theologisches Werk, die Selbstständigkeit gegenüber den üblichen Gedankengängen der Sentenzen, die danach streben, sich in der Lehre zu behaupten, machen dazu aus ihm ein nützliches Dokument für die Erkenntnis einer Epoche des Übergangs, für die man nur mehr Dokumente besitzen wünschte, die die Atmosphäre verstehen lassen, in der sich das Zeitalter der Hochscholastik vorbereitete.“ 